PROCEEDINGS OF THE

AMERICAN MATHEMATICAL SOCIETY

Volume 128, Number 4, Pages 1203-1206

S 0002-9939(99)05249-1

Article electronically published on July 28, 1999

\title{
WEAK ERGODICITY OF STATIONARY PAIRWISE INDEPENDENT PROCESSES
}

\author{
D. LANDERS AND L. ROGGE
}

(Communicated by James Glimm)

\begin{abstract}
It is proven that a stationary process of pairwise independent random variables with values in a separable metric space is weakly ergodic, i.e. each random variable is independent of the system of invariant sets of the process. An example shows that a process of identically distributed pairwise independent random variables is in general, however, not weakly ergodic.
\end{abstract}

\section{INTRODUCTION}

Two famous results of probability theory for pairwise independent random variables, namely Etemadi's strong laws of large numbers (see [6], [7]) and the BorelCantelli Lemma (see [3, Th. 4.2.5), led to an increasing interest in the study of pairwise independent random variables. Unfortunately, however, it turned out that pairwise independence performs poorly as a substitute for independence: It was shown that neither the central limit theorem nor the law of the iterated logarithm nor Kolmogoroff's zero-one law hold for pairwise independent instead of independent random variables (see [1, [5], [8], [9]).

Cuesta and Matrán constructed a stationary process of pairwise independent random variables which simultaneously violates the just cited three famous laws of probability theory (see [5]). Their nice and tricky example also shows that for a stationary process of pairwise independent random variables the zero-one law does not hold for the invariant sets; i.e. such a process is not ergodic in general. In Theorem 2.1 we prove that such a process is, however, weakly ergodic; a result which is not true any more for identically distributed pairwise independent random variables (see Example 2.2).

\section{THE RESULtS}

Let $(\Omega, \mathcal{A}, P)$ be a probability space and $(M, \delta)$ a separable metric space with Borel $\sigma$-field $\mathcal{B}$. Let $X_{n}: \Omega \rightarrow M, n \in \mathbb{N}$, be random variables, i.e. $\mathcal{A}, \mathcal{B}$-measurable functions. $X_{n}, n \in \mathbb{N}$, is called a stationary process if the distribution of $\left(X_{k}, X_{k+1}\right.$, $\ldots)$ is the same for all $k \in \mathbb{N}$. We denote by $\mathcal{I}\left(X_{n}, n \in \mathbb{N}\right)$ the $\sigma$-field of all invariant sets of the process $X_{n}, n \in \mathbb{N}$; i.e. $I \in \mathcal{I}\left(X_{n}, n \in \mathbb{N}\right)$ iff $I=\left(X_{n}\right)_{n \in \mathbb{N}}^{-1}(B)$ where $B \subset M^{\mathbb{N}}$ is an invariant Borel set, that means $B \in \mathcal{B}^{\mathbb{N}}$ and $\left(x_{1}, x_{2}, \ldots\right) \in B \Longleftrightarrow$

Received by the editors May 19, 1998 .

1991 Mathematics Subject Classification. Primary 60G10; Secondary 60F20.

Key words and phrases. Stationary processes, pairwise independent random variables, ergodicity.

(C)2000 American Mathematical Society 
$\left(x_{2}, x_{3}, \ldots\right) \in \mathcal{B}$. The process $X_{n}, n \in \mathbb{N}$, is called ergodic, if $P(I) \in\{0,1\}$ for all $I \in \mathcal{I}\left(X_{n}, n \in \mathbb{N}\right)$ (see e.g. [2], pp. 118). $X_{n}, n \in \mathbb{N}$, is called weakly ergodic, if each $X_{k}$ is independent of $\mathcal{I}\left(X_{n}, n \in \mathbb{N}\right)$. Observe that each ergodic process is weakly ergodic, but not conversely. Ergodic processes have been widely investigated in the literature, and we hope that the new concept of weak ergodic processes will be similarly useful.

2.1 Theorem. Let $X_{n}, n \in \mathbb{N}$, be a stationary process of pairwise independent random variables with values in a separable metric space $M$. Then $X_{n}, n \in \mathbb{N}$, is weakly ergodic.

Proof. Since a separable metric space can be considered as a subspace of an uncountable, complete and separable metric space, we can, without loss of generality, assume that $M$ is already an uncountable, complete and separable metric space. Hence there exists a Borel-isomorphism $\psi: M \rightarrow \mathbb{R}$ (see Theorem 8.3.6 of [4]). Then $Y_{n}:=\psi \circ X_{n}, n \in \mathbb{N}$, is a stationary process of pairwise independent realvalued random variables. Since $\mathcal{I}\left(Y_{n}, n \in \mathbb{N}\right)=\mathcal{I}\left(X_{n}, n \in \mathbb{N}\right)$ (see Lemma 2.3 (ii)), it suffices to show that $Y_{n}, n \in \mathbb{N}$, is weakly ergodic. Let $k \in \mathbb{N}$ and $x \in \mathbb{R}$ be fixed. It suffices to show that

$$
\left\{Y_{k} \leq x\right\} \text { and } \mathcal{I}\left(Y_{n}, n \in \mathbb{N}\right) \text { are independent. }
$$

Let $\varphi: \mathbb{R} \rightarrow] 0,1\left[\right.$ be injective and Borel-measurable. Then $\varphi \circ Y_{n}, n \in \mathbb{N}$, is a stationary process of pairwise independent random variables. Hence we have by the ergodic theorem (see Theorem 6.28 of [2])

$$
\frac{1}{n} \sum_{i=1}^{n} \varphi \circ Y_{i} \rightarrow E\left(\varphi \circ Y_{k} \mid \mathcal{I}\left(\varphi \circ Y_{n}, n \in \mathbb{N}\right)\right) \text { a.e. }
$$

Furthermore there holds by the theorem of Etemadi (see [6])

$$
\frac{1}{n} \sum_{i=1}^{n} \varphi \circ Y_{i} \rightarrow E\left(\varphi \circ Y_{k}\right) \text { a.e. }
$$

By (2) and (3) we obtain

$$
E\left(\varphi \circ Y_{k}\right)=E\left(\varphi \circ Y_{k} \mid \mathcal{I}\left(\varphi \circ Y_{n}, n \in \mathbb{N}\right)\right) \text { a.e. }
$$

Since $\mathcal{I}\left(\varphi \circ Y_{n}, n \in \mathbb{N}\right)=\mathcal{I}\left(Y_{n}, n \in \mathbb{N}\right)=: \mathcal{I}$ by Lemma 2.3 (ii), we obtain that

$$
E\left(\varphi \circ Y_{k}\right)=E\left(\varphi \circ Y_{k} \mid \mathcal{I}\right) \text { a.e. }
$$

It is easy to see that there exists a sequence of injective and Borel-measurable functions $\left.\varphi_{n}: \mathbb{R} \rightarrow\right] 0,1\left[\right.$ with $\varphi_{n}(t) \underset{n \rightarrow \infty}{\rightarrow} 1_{]-\infty, x]}(t)$ for all $t \in \mathbb{R}$. Hence we obtain by (4) for all $n \in \mathbb{N}$

$$
E\left(\varphi_{n} \circ Y_{k}\right)=E\left(\varphi_{n} \circ Y_{k} \mid \mathcal{I}\right) \text { a.e. }
$$

By Lebesgue's dominated convergence theorem (5) yields

$$
E\left(1_{]-\infty, x]} \circ Y_{k}\right)=E\left(1_{]-\infty, x]} \circ Y_{k} \mid \mathcal{I}\right) \text { a.e., }
$$

which implies (1).

The following example is a special case of example 2.3 of [5] adapted to our special need. 
2.2 Example. There exists a sequence of real-valued pairwise independent and identically distributed random variables $X_{n}, n \in \mathbb{N}$, such that $X_{n}, n \in \mathbb{N}$, is not weakly ergodic.

Proof. Let $X_{2 n-1}, n \in \mathbb{N}$, be independent $\{0,1\}$-valued random variables with $P\left(X_{2 n-1}=0\right)=P\left(X_{2 n-1}=1\right)=1 / 2$. Put

$$
X_{2 n}:=X_{2 n+1} \oplus X_{1}
$$

where $\oplus$ means sum mod 2. Then it is easy to see that $X_{n}, n \in \mathbb{N}$, are pairwise independent and identically distributed. We show that there exists $I \in \mathcal{I}\left(X_{n}, n \in \mathbb{N}\right)$ with

$$
P\left(\left\{X_{1}=0\right\} \triangle I\right)=0,
$$

where $A \triangle B=(A \backslash B) \cup(B \backslash A)$.

Since $P\left(\left\{X_{1}=0\right\}\right)=1 / 2,(1)$ implies that $I$ is not independent of $\left\{X_{1}=0\right\}$; hence $X_{n}, n \in \mathbb{N}$, is not weakly ergodic. To prove (1) put

$$
I:=\left\{\varlimsup \frac{1}{n} \sum_{i=2}^{n+1} 1_{\left\{X_{i}=X_{i+1}\right\}}>1 / 2\right\} .
$$

If $X_{1}(\omega)=0$, then $X_{2 k}(\omega)=X_{2 k+1}(\omega)$ and $X_{2 k-1}(\omega)=X_{2 k}(\omega) \Longleftrightarrow X_{2 k-1}(\omega)=$ $X_{2 k+1}(\omega)$. Hence for $X_{1}(\omega)=0$ we have

$$
\begin{aligned}
Y_{n} & :=\frac{1}{n} \sum_{i=2}^{n+1} 1_{\left\{X_{i}=X_{i+1}\right\}} \\
& =\frac{1}{n} \#\{k: 2 \leq 2 k \leq n+1\}+\frac{1}{n} \sum_{2 \leq 2 k-1 \leq n+1} 1_{\left\{X_{2 k-1}=X_{2 k+1}\right\}} .
\end{aligned}
$$

As $\left\{X_{2 k-1}=X_{2 k+1}\right\}, k \in \mathbb{N}$, are independent with $P\left\{X_{2 k-1}=X_{2 k+1}\right\}=1 / 2$, we obtain by the strong law of large numbers that $Y_{n} \rightarrow 3 / 4 \quad P$-a.e. Therefore

$$
\left\{X_{1}=0\right\} \subset I \quad P \text {-a.e. }
$$

Since furthermore $X_{1}(\omega)=1$ implies $X_{2 k}(\omega) \neq X_{2 k+1}(\omega)$, we obtain $\left\{X_{1}=1\right\} \subset$ $\Omega \backslash I$. This and (3) imply (1).

2.3 Lemma. Let $M, M_{1}$ be separable metric spaces. Let $X_{n}, n \in \mathbb{N}$, be random variables with values in $M$.

(i) If $\psi: M \rightarrow M_{1}$ is Borel-measurable, then

$$
\mathcal{I}\left(\psi \circ X_{n}, n \in \mathbb{N}\right) \subset \mathcal{I}\left(X_{n}, n \in \mathbb{N}\right) .
$$

(ii) If $\psi: M \rightarrow \mathbb{R}$ is injective and Borel-measurable and if furthermore $M$ is complete, then

$$
\mathcal{I}\left(\psi \circ X_{n}, n \in \mathbb{N}\right)=\mathcal{I}\left(X_{n}, n \in \mathbb{N}\right) .
$$

Proof. (i) Put $\Phi\left(x_{1}, x_{2}, \ldots\right):=\left(\psi\left(x_{1}\right), \psi\left(x_{2}\right), \ldots\right)$. Then $\Phi: M^{\mathbb{N}} \rightarrow M_{1}^{\mathbb{N}}$ is Borelmeasurable. Let $I \in \mathcal{I}\left(\psi \circ X_{n}, n \in \mathbb{N}\right)$. Then there exists an invariant Borel set $B \subset M_{1}^{\mathbb{N}}$ with

$$
I=\left(X_{n}\right)_{n \in \mathbb{N}}^{-1}\left(\Phi^{-1}(B)\right) .
$$

As $\Phi^{-1}(B)$ is an invariant Borel set of $M^{\mathbb{N}}$, we obtain $I \in \mathcal{I}\left(X_{n}, n \in \mathbb{N}\right)$. 
(ii) According to (i) it suffices to show

$$
\mathcal{I}\left(X_{n}, n \in \mathbb{N}\right) \subset \mathcal{I}\left(\psi \circ X_{n}, n \in \mathbb{N}\right) .
$$

This follows from (i) applied to $\psi \circ X_{n}$ instead of $X_{n}$ and $\psi^{-1}: \psi(M) \rightarrow M$ instead of $\psi$, as $\psi^{-1}$ is Borel-measurable (see Theorem 8.3.7 and Proposition 8.3.5 of [3]).

We thank one of the referees for valuable suggestions.

\section{REFERENCES}

[1] Richard C. Bradley, A stationary, pairwise independent, absolutely regular sequence for which the central limit theorem fails, Probability Theory and Rel. F. 81 (1989), 1-10. MR 90b:60023

[2] Leo Breiman, Probability, Addison Wesley Publishing Company, Reading and London, 1968. MR 37:4841

[3] K.L. Chung, A course in probability theory, Academic Press, Orlando, Florida, 1974. MR 49:11579

[4] Donald L. Cohn, Measure theory, Birkhäuser, Boston and Stuttgart, 1980. MR 81k:28001

[5] Juan A. Cuesta and Carlos Matrán, On the asymptotic behavior of sums of pairwise independent random variables, Statistics and Prob. Lett. 11 (1991), 201-210. MR 92h:60023a

[6] Nasrollah Etemadi, An elementary proof of the strong law of large numbers, Z. Wahrsch. Verw. Geb. 55 (1981), 119-122. MR 82b:60027

[7] Nasrollah Etemadi, On the laws of large numbers for nonnegative random variables, J. Multivariate Anal. 13 (1983), 187-193. MR 85d:60062

[8] Svante Janson, Some pairwise independent sequences for which the central limit theorem fails, Stochastics 23 (1988), 439-448. MR 89e:60048

[9] A. Joffe, On a sequence of almost deterministic pairwise independent random variables, Proc. Amer. Math. Soc. 29 (1971), 381-382. MR 43:5578

Mathematisches Institut der Universität Zu Köln, Weyertal 86, D-50931 Köln, GerMANY

E-mail address: landers@mi.uni-koeln.de

Fachbereich Mathematik der Gerhard-Mercator-Universität ghs Duisburg, Lotharstr. 65, D-47048 Duisburg, Germany

E-mail address: rogge@math.uni-duisburg.de 